\title{
A Multichamber Fluidic Device for 3D Cultures Under Interstitial Flow With Live Imaging: Development, Characterization, and Applications
}

\author{
Carmen Bonvin, ${ }^{1}$ Jan Overney, ${ }^{1}$ Adrian C. Shieh, ${ }^{1,2}$ J. Brandon Dixon, ${ }^{1,2}$ \\ Melody A. Swartz ${ }^{1,2}$ \\ ${ }^{1}$ Institute of Bioengineering, School of Life Sciences/LMBM/Station 15, \\ Ecole Polytechnique Fédérale de Lausanne (EPFL), Lausanne 1015, Switzerland \\ ${ }^{2}$ Department of Biomedical Engineering, Northwestern University, Evanston, Illinois; \\ telephone: 41-21-693-9686; fax: 41-21-693-1660; e-mail: melody.swartz@epfl.ch
}

Received 16 July 2009; revision received 9 0ctober 2009; accepted 9 November 2009

Published online 1 December 2009 in Wiley InterScience (www.interscience.wiley.com). DOI 10.1002/bit.22608

\begin{abstract}
Interstitial flow is an important biophysical cue that can affect capillary morphogenesis, tumor cell migration, and fibroblast remodeling of the extracellular matrix, among others. Current models that incorporate interstitial flow and that are suitable for live imaging lack the ability to perform multiple simultaneous experiments, for example, to compare effects of growth factors, extracellular matrix composition, etc. We present a nine-chamber radial flow device that allows simultaneous 3D fluidic experiments for relatively long-term culture with live imaging capabilities. Flow velocity profiles were characterized by fluorescence recovery after photobleaching (FRAP) for flow uniformity and estimating the hydraulic conductivity. We demonstrate lymphatic and blood capillary morphogenesis in fibrin gels over 10 days, comparing flow with static conditions as well as the effects of an engineered variant of VEGF that binds fibrin via Factor XIII. We also demonstrate the culture of contractile fibroblasts and co-cultures with tumor cells for modeling the tumor microenvironment. Therefore, this device is useful for studies of capillary morphogenesis, cell migration, contractile cells like fibroblasts, and multicellular cultures, all under interstitial flow.

Biotechnol. Bioeng. 2010;105: 982-991.

(C) 2009 Wiley Periodicals, Inc.
\end{abstract}

KEYWORDS: FRAP; capillary morphogenesis; fibroblast; lymphatic; biomechanics; tissue engineering

\section{Introduction}

Tissue engineering unifies many different design principles toward the ultimate goal of recreating functional living

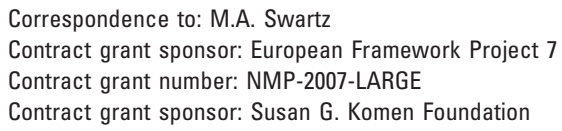

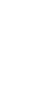


remodel the extracellular matrix to maintain a desired mechanical environment (Pedersen and Swartz, 2005); in this way, if the pressure gradient remains fixed, cellmediated changes in matrix architecture could lead to altered flow rates, allowing one to mimic the physiological environment that drives matrix remodeling using fixed pressure-driven flows.

Given the importance of interstitial flow in these processes, a number of platforms have been developed to generate interstitial flow in fluidic devices ( $\mathrm{Ng}$ and Pun, 2008; Vickerman et al., 2008; Wang and Tarbell, 2000). However, many of these systems lack the throughput necessary to examine and compare various conditions that could be of interest to tissue engineers trying to recapitulate functional microvascular networks. In angiogenesis assays, for example, it may be important to compare multiple conditions simultaneously, such as different combinations of cells, growth factors, biomaterials, etc. Development of a fluidic system that could allow multiple samples under different conditions to be run side-by-side would represent an important new tool for such studies.

We present a system incorporating nine identical chambers, allowing direct comparison of different experimental conditions exposed to the same interstitial flow microenvironment. This system allows cell culture in a 3D environment, mimicking the tissue microenvironment to study cell-cell, cell-matrix, and cell-flow behavior in vitro. In addition to the multiple chambers, this device incorporates a number of key features, including (1) small size to reduce diffusional barriers through the gel, (2) control over the pressure gradient, (3) anchored matrix to prevent contraction, (4) live and post-fixation imaging via multiple microscopy modalities, and (5) the possibility to isolate the gel for post-experimental analysis. The system was validated by comparing the effects of interstitial flow and matrix-bound VEGF on BEC and LEC morphogenesis, by culturing fibroblasts to demonstrate prevention of matrix contraction, and with co-cultures of tumor cells and fibroblasts.

\section{Methods}

\section{Device Design and Fabrication}

The multichamber radial flow system consists of nine identical chambers sharing inlet and outlet medium reservoirs (Fig. 1). Polydimethylsiloxane (PDMS) was chosen for its biocompatibility as well as its good optical properties. A glass coverslip $(60 \mathrm{~mm}$ diameter, thickness no. 1, VWR, Dietikon, Switzerland) constitutes the bottom surface of the system to allow light microscopy, and hydrophilic porous polyethylene $(\mathrm{PE})$ rings are inserted between the PDMS and glass to anchor the matrix and prevent matrix contraction by the embedded cells. The same pressure gradient is applied across all nine wells allowing homogeneous radial flow (whose velocity decays radially

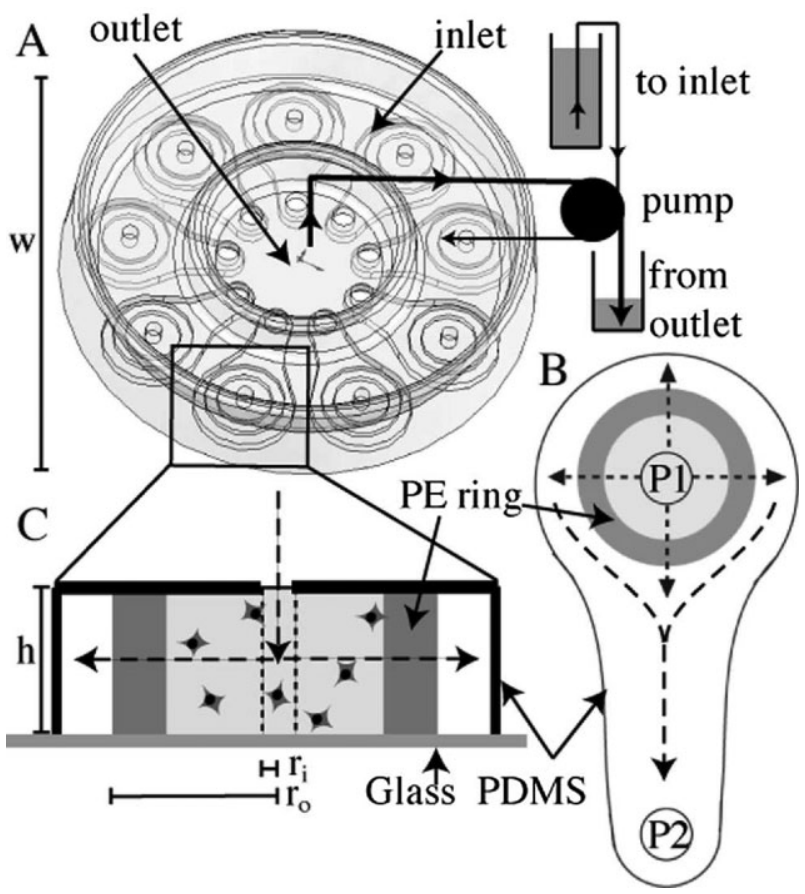

Figure 1. The nine-chamber radial flow system. A: The system is made of a single polydimethylsiloxane (PDMS) piece that defines the chambers and medium reservoirs, and sits atop a glass coverslip. A pump brings media to the inlet reservoir and removes media from the outlet reservoir to maintain the pressure drop. B: Cells are seeded in the nine individual compartments and porous polyethylene (PE) rings confine the gel and prevents contraction. Culture medium is added to the inlet reservoir, causing the inlet pressure head (P1) to be higher than the outlet pressure (P2) for all chambers to drive flow through the 3D cell-gel compartment. C: Side view of one chamber. Arrows indicate flow direction. Dimensions are as follows: $h=1 \mathrm{~mm}$; $w=50 \mathrm{~mm} ; r_{i}=0.7 \mathrm{~mm} ; r_{0}=4.05 \mathrm{~mm}$; thickness of PE ring $=1.1 \mathrm{~mm}$.

according to the geometry of the system), since the only path for fluid flow from the inlet to the outlet reservoir is through the gel-filled chamber (Fig. 1). To create the PDMS component, a mold was micromachined out of polymethylmethacrylate (PMMA). PDMS (Dow Corning, Wiesbaden, Germany 184 Sylgard base and curing agent, 10:1 ratio) was injected into the mold and polymerized at $90^{\circ} \mathrm{C}$ for $90 \mathrm{~min}$. The PE rings, with inner and outer radii of 2.95 and $4.05 \mathrm{~mm}$, respectively, were laser cut from a $1.6 \mathrm{~mm}$ thick sheet of PE (Small Parts PEH-060/90, Miramar, FL). The width of the system $(50 \mathrm{~mm})$ was chosen to allow mounting on a circular glass coverslip with a diameter of $60 \mathrm{~mm}$. Nine chambers were used to fit the most chambers on one slide while maintaining as much PDMS surface to allow proper bonding to the glass. Each chamber had a height of $1 \mathrm{~mm}$ and an inlet radius of $0.7 \mathrm{~mm}$.

\section{System Assembly and Filling}

Before assembling, the PDMS and PE components were autoclaved and the glass coverslip was cleaned with ethanol and then acetone. PDMS is hydrophobic, but can be 
temporarily rendered more hydrophilic to facilitate filling by oxygen plasma treatment ( $200 \mathrm{~W}, 0.3$ Torr vacuum, $60 \mathrm{~s}$ ) (Branson/IPC, Danbury, CT, Series 2000 Plasma processing reactor center). Following treatment, the PDMS and the glass were pressed together to bond the two components. The chambers were further treated to facilitate surface bonding with the gel, first with poly-L-lysine $(0.01 \%$, Sigma, Steinheim, Germany) for $25 \mathrm{~min}$ and then functionalized with $0.1 \%$ glutaraldehyde (Fluka, Buchs, Switzerland) for $25 \mathrm{~min}$. After treatment, the assembled system was rinsed thoroughly with deionized water and stored at $4{ }^{\circ} \mathrm{C}$ with all chambers filled with water for 1-3 days.

The cell-containing biomatrices (prepared as described below) were injected into each chamber at $40 \mu \mathrm{L}$ each and allowed to polymerize at $37^{\circ} \mathrm{C}$ for $20 \mathrm{~min}$. Culture medium was added to both the central and outer medium reservoirs for static conditions, and left overnight prior to application of flow. To initiate flow, medium was removed from the central (outlet) reservoir, creating a pressure gradient of 0.2 , 0.3 , or $0.5 \mathrm{~cm} \mathrm{H}_{2} \mathrm{O}$ across each well. The magnitude of the pressure gradient could be varied by adjusting the amount of medium in the outer (inlet) reservoir. The pressure head was held constant during the experiment by a pump, which brings culture medium to the inlet reservoir and removes any excess from the outlet reservoir. Furthermore, the inlet reservoir was taller than the outlet reservoir, allowing excess media to overflow from the inlet to the outlet; thus, by setting the pump to a higher flow rate for the outlet tubing, or by choosing a larger diameter tube, the outlet reservoir can be kept empty.

\section{Flow Characterization}

Fluorescence recovery after photobleaching (FRAP) was used to determine flow through the gel, following methods previously described (Berk et al., 1996). The radial flow system was placed in a glass-bottomed Petri dish and the collagen gel was perfused with a solution of $100 \mu \mathrm{g} / \mathrm{mL}$ of $40 \mathrm{kDa}$ FITC-conjugated dextran (Sigma). To determine the flow velocities throughout the chamber (see below), five equally spaced measurements were made along four orthogonal radii in each chamber. The region of interest was a $90 \mu \mathrm{m}$ circle in the center of the image. Once selected, it was bleached by performing 100 scans at 5 frames per second (fps) across the bleaching region with a $488 \mathrm{~nm}$, $35 \mathrm{~mW}$ argon krypton laser, using an inverted Zeiss LSM 510 Meta confocal microscope. Sequential images were taken after bleaching to image the collective effects of diffusion and convection on the distribution of the bleached area. A customized algorithm was written in Matlab (MathWorks, Natick, MA) to extract the displacement of the bleached area due to convection. Specifically, a low-pass filter and threshold was applied to the image of the original bleached area and the image was cropped around the centroid of the bleached area such that the entire field of view was $4 a \times 4 a$ pixels where $a$ is the radius of the bleached spot. This crop was applied to each subsequent image followed by a twodimensional discrete fast Fourier transform (FFT). Since a phase shift in the frequency domain corresponds to a displacement in the spatial domain, and since the velocity can be considered constant over the time course of the measurement, the fluid velocity vector could be calculated from the following equations:

$$
\begin{gathered}
x_{i}=\frac{W}{2 \pi} \cos ^{-1}\left[\frac{\operatorname{Re}\left(I_{x, 0}\right)}{\sqrt{\operatorname{Re}\left(I_{x, 0}\right)^{2}+\operatorname{Im}\left(I_{x, 0}\right)^{2}}}\right] \\
y_{i}=\frac{H}{2 \pi} \cos ^{-1}\left[\frac{\operatorname{Re}\left(I_{y}\right)}{\sqrt{\operatorname{Re}\left(I_{0, y}\right)^{2}+\operatorname{Im}\left(I_{0, y}\right)^{2}}}\right] \\
v_{x}=\frac{n \sum_{1}^{i} x_{i} t_{i}-\sum_{1}^{i} x_{i} \sum_{1}^{i} t_{i}}{n \sum_{1}^{i} x_{i}^{2}-\left(\sum_{1}^{i} x_{i}\right)} \\
v_{y}=\frac{n \sum_{1}^{i} y_{i} t_{i}-\sum_{1}^{i} y_{i} \sum_{1}^{i} t_{i}}{n \sum_{1}^{i} y_{i}^{2}-\left(\sum_{1}^{i} y_{i}\right)^{2}}
\end{gathered}
$$

where $v_{x}$ and $v_{y}$ are the $x$ and $y$ components of the velocity, $x_{i}$ and $y_{i}$ are the phases of the first harmonic for each image $i, I_{x, 0}$ and $I_{0, y}$ are the complex components of the first harmonic of the FFT in $x$ and $y$, respectively; $W$ and $H$ are the width and height of the cropped image, and $t_{i}$ is the time at which each image $i$ was captured.

The bulk-averaged velocity should decrease with increasing radius $r$ according to conservation of mass:

$$
\bar{v}=\frac{Q}{A}=\frac{Q}{2 \pi r h}
$$

where $Q$ is the total flow rate through one chamber (estimated by the slope of $1 / r$ vs. velocity), $A$ is the area normal to flow, and $h$ is the gel height. Then, using Darcy's law

$$
\bar{v}=-\frac{K^{\prime}}{\mu} \nabla P
$$

where $K^{\prime}$ is the hydraulic conductivity of the gel, $\mu$ is the viscosity of the medium $(\sim 0.75 \mathrm{cp}$ for $20 \%$ serumcontaining endothelial cell medium at $37^{\circ} \mathrm{C}$ (Helm et al., $2005))$, and $\nabla P$ is the imposed pressure gradient $(=\mathrm{d} P / \mathrm{d} r)$. We can integrate over the radius and estimate $K^{\prime}$ from the slope of $\Delta P$ versus $Q$ :

$$
Q=\frac{2 \pi h}{\mu \ln \left(r_{\text {outlet }} / r_{\text {inlet }}\right)} K^{\prime} \Delta P
$$

where $\Delta P=P_{\text {inlet }}-P_{\text {outlet }}\left(=0.2,0.3\right.$, and $0.5 \mathrm{~cm} \mathrm{H}_{2} \mathrm{O}$ in these example experiments); $r_{\text {outlet }}$ is the outer chamber 
radius $(4.05 \mathrm{~mm})$, and $r_{\text {inlet }}$ is the radius of the flow inlet $(0.7 \mathrm{~mm})$. Since all chambers are exposed to the same pressure gradient and the inlet is centered in the circular PE ring, we expect the bulk fluid velocity to be uniform at a given radius and consistent between different chambers that have similar hydraulic conductivities $K^{\prime}$.

\section{Cells}

For capillary morphogenesis demonstrations, human dermal microvascular lymphatic and blood endothelial cells were isolated as described (Yong et al., 2005) and expanded in collagen-coated flasks in endothelial cell basal medium (EBM) supplemented with 20\% FBS, 1\% penicillin-streptomycin-amphotericin B (PSA), hydrocortisone, and cyclic AMP (all from Lonza, Basel, Switzerland) and maintained at $5 \% \mathrm{CO}_{2}$ and $37^{\circ} \mathrm{C}$. They were used at passage 6-8. In some experiments, a recombinant variant of VEGF $_{121}$ that cross-links into fibrin, $\alpha_{2} \mathrm{PI}_{1-8}-\mathrm{VEGF}_{121}$ (Helm et al., 2007; Zisch et al., 2001) (kind gift of Andreas Zisch), was added to the fibrin before polymerization at $100 \mathrm{ng} / \mathrm{mL}$.

For fibroblast-tumor cell co-cultures, both human and mouse cells were tested in two different schematics. Human dermal fibroblasts were isolated from the same foreskins used for the LEC and BEC cell isolation by negative selection, cultured in DMEM high glucose medium (PAA, Pasching, Austria) supplemented with 10\% FBS and 1\% PSA and used at passages 7-10. For visualization within the chambers, cells were labeled with Cell Tracker Red (Invitrogen, Basel, Switzerland). GFP-labeled MDA-MB435S human melanoma cells (described in (Skobe et al., 2001)) were a kind gift of Mihaela Skobe, and were expanded in DMEM high glucose supplemented with 20\% FBS and 1\% PSA.

In the second scenario, mimicking invasion of tumor cells into a fibroblast-seeded gel, $\mathrm{GFP}^{+}$murine B16-F10 melanomas (ATCC, Middlesex, UK) and tomato protein ${ }^{+}$ lymph node T-cell area associated reticular fibroblasts (TRCs, kind gift of Sanjiv Luther) were used. The melanoma cells were transduced with a PGK-GFP lentiviral vector (kind gift of Didier Trono); stably transduced cells were FACS sorted for a high expression of GFP and expanded in DMEM supplemented with 10\% FBS and 1\% PSA. The TRCs are described elsewhere (Tomei et al., 2009). They were cultured in RPMI 1640 (Gibco, Invitrogen, Basel, Switzerland) supplemented with Glutamax (Gibco), 10\% FBS, $1 \%$ PSA, HEPES (12 nM), and $\beta$-mercaptoethanol (50 $\mathrm{MM}$, Sigma).

\section{Gel Preparation}

For the endothelial cell cultures, fibrin gels were used, consisting of $3 \mathrm{mg} / \mathrm{mL}$ human fibrinogen, $200 \mathrm{U} / \mathrm{mL}$ aprotinin, and $2 \mathrm{U} / \mathrm{mL}$ human thrombin (all from Sigma); 2 U/mL factor XIII (Baxter, Deerfield, IL, 2 mM
$\mathrm{CaCl}_{2}$ (Fisher), and $100 \mathrm{ng} / \mathrm{mL} \quad \alpha_{2} \mathrm{PI}_{1-8}-\mathrm{VEGF}_{121}$ (where indicated), in TBS (Helm et al., 2007). Cells were mixed at $1.5 \times 10^{6}$ cells $/ \mathrm{mL}$ before pipetting into the chambers. For the FRAP experiments, $1.8 \mathrm{mg} / \mathrm{mL}$ type I collagen (BD Biosciences, Allschwil, Switzerland) was used to permit comparison of measured Darcy permeabilities with published data (Ng and Swartz, 2003). For the tumor-fibroblast co-cultures, $90 \%$ type I collagen $(2.5 \mathrm{mg} / \mathrm{mL})$ was mixed with 10\% Matrigel (BD Biosciences) and 250,000 cells $/ \mathrm{mL}$ (fibroblasts) with 500,000 cells/mL (tumor cells) (in the first scenario) or $10^{6}$ cells $/ \mathrm{mL}$ (fibroblasts) with later added injection of $2.8 \times 10^{6}$ cells $/ \mathrm{mL}$ tumor cells. The mixtures were pipetted into the chamber and incubated overnight before starting flow.

\section{Imaging}

To image cell structure and organization, the cells were stained for actin fibers with Alexa Fluor 488 phalloidin (Invitrogen). The cells were fixed using 2\% paraformaldehyde by filling the reservoirs for $1 \mathrm{~h}$ at $4{ }^{\circ} \mathrm{C}$ and then stained overnight at $4^{\circ} \mathrm{C}$ with $5 \mathrm{U} / \mathrm{mL}$ phalloidin. The fibrin matrix was visualized via confocal reflectance microscopy. The gels were imaged on an inverted confocal microscope (Leica SP5) and 2D projections of the 3D stacks were generated, which were further processed using ImageJ (NIH publicdomain image processing software). Cell viability was tested using a Live/Dead cell staining kit (Invitrogen).

\section{Results}

The key feature of this device is its multiple chambers that allow nine simultaneous experiments to be performed; for example, for different matrices, cell concentrations, cell types, etc. For this reason, all chambers were created in a single PDMS unit and feed from a common inlet reservoir and flow into a common outlet reservoir. Since all chambers shared the same reservoirs, the fluid pressure drop across each is the same, leading to a similar flow velocity in each case providing that the resistance is the same in each gel ( since velocity is inversely related to $K^{\prime}$ ). The nine-chamber radial flow system was based on a previously developed radial flow chamber ( $\mathrm{Ng}$ and Swartz, 2003, 2006; $\mathrm{Ng}$ et al., $2004,2005)$. Nine smaller versions of the single chamber fit onto a system with a total diameter of $5 \mathrm{~cm}$, which can be placed in a traditional Petri dish and mounted on a microscope stage, and each chamber holds $40 \mu \mathrm{L}$, significantly reducing the amount of reagents and the number of cells used. The PE rings are critical for confining the gel to a delimited region and necessary to prevent gel contraction by contractile cells (Kolodney and Wysolmerski, 1992). Since the hydraulic conductivity of PE is far greater than that of the fibrin or collagen gels used here ( $\mathrm{Ng}$ and Swartz, 2003), the PE ring does not contribute to the hydraulic resistance of the system. 


\section{Flow Profile}

The radial decay in velocity within the gel was confirmed quantitatively with FRAP (Fig. 2A). We found that the flow was uniform along all axes, decayed with radius (Fig. 2B), and was consistent between chambers at a given radius. We also confirmed that the velocity at any point increased linearly with increasing pressure head according to Darcy's law, and that the flow rate increased linearly with pressure (Fig. 2C). From the fit of the data to Eq. (6), we determined the Darcy permeability of the $1.8 \mathrm{mg} / \mathrm{mL}$ type I collagen gel to be $K^{\prime}=4 \times 10^{-10} \mathrm{~cm}^{2}$, which is within previously measured values ( $\mathrm{Ng}$ and Swartz, 2003). The average velocity in collagen was higher than in fibrin, consistent with the lower concentration of collagen $(1.8 \mathrm{mg} / \mathrm{mL}$ vs. $3 \mathrm{mg} / \mathrm{mL}$ fibrin) and cross-linked nature of fibrin. It is important to note however that the velocity values greatly depend on the experimental setup (e.g., matrix concentration, cell type, length of experiment, etc.).

To address the issue of possible cross-contamination of cell-secreted factors between wells, we loaded alternating wells with $100 \mu \mathrm{g} / \mathrm{mL}$ of $10 \mathrm{kDa}$ rhodamine-dextran mixed within the collagen gels and imaged wells at different times under static versus flow conditions (Fig. 2D). We found very little fluorescence contamination in neighboring wells under static conditions at 1 or 5 days, and could detect none in flow conditions, which was consistent with the estimated Peclet number between the neighboring well outlets being greater than unity. We note that the fluorescence was more quickly flushed out in flow versus static conditions, but even in static conditions it decayed over 5 days.

To further validate our model, we tested two different processes: capillary morphogenesis and tumor invasion. The purpose of the first was to show reproducibility of previous data using this new system, while the purpose of the second was to show the versatility of the device in the types of cells used or the seeding scheme.

\section{Capillary Morphogenesis Under Flow and $\alpha_{2} \mathrm{PI}_{1-8}-$ VEGF $_{121}$}

We compared static versus flow conditions on LECs, as flow was previously shown to promote lymphatic capillarogenesis (Boardman and Swartz, 2003; Ng et al., 2004), as well as the effects of matrix-bound VEGF on LEC and BEC morphogenesis under flow (Helm et al., 2005) for 10 days. We found that interstitial flow of $1-2 \mu \mathrm{m} / \mathrm{s}$ induced from a $5 \mathrm{~mm}$ pressure drop (Fig. 2B) drove organization of LECs during the 5- and 10-day experiments. In an experiment comparing our previous single chamber system $(\mathrm{Ng}$ and Swartz, 2003) and the nine-chamber system, LECs grew, responded to flow, and organized in a similar manner (Fig. 3A,B). In both cases, when cells were grown in the static condition, they remained rounded within the gel, while when exposed to flow, they spread and formed multicellular structures containing lumen (Fig. 3A). Viability within the chamber was similar between a traditional culture (control)
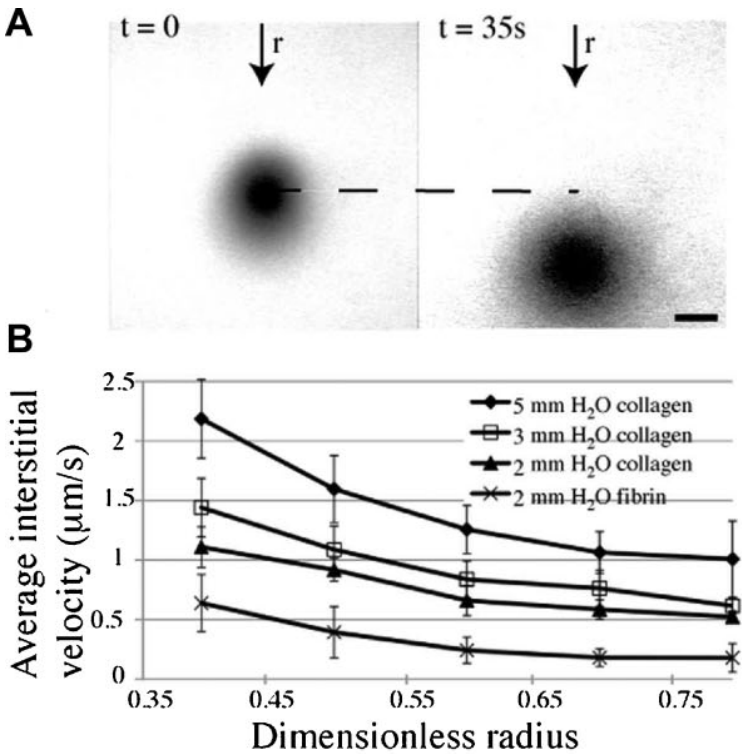

C

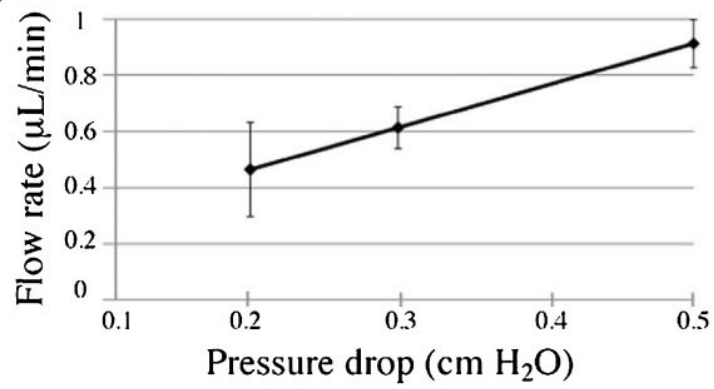

D

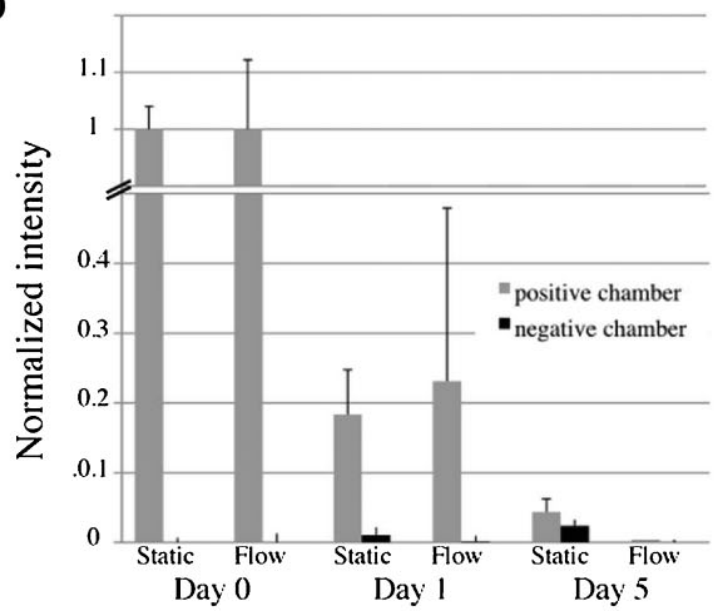

Figure 2. Characterization of interstitial flow within collagen or fibrin gels in the 9-chamber flow system using FRAP. A: A circular region of interest is photobleached (left) and tracked for $35 \mathrm{~s}$ (right). Scale bar: $100 \mu \mathrm{m}$. B: Velocity as a function of radius follows the expected decline, and increases linearly with pressure. Each point represents averages from data taken in each quadrant of the chamber and in different chambers. The radial position and pressure drop had a significant influence on flow velocity $(P<0.05$ using ANOVA). C: From the collagen data represented in (B), the flow increases linearly as a function of the pressure head as expected. The slope represents the hydraulic conductivity $K$, which in this case is $4 \times 10^{-10} \mathrm{~cm}^{2}$ for collagen. D: Diffusion of $10 \mathrm{kDa}$ rhodamine-dextran from a chamber initially filled with fibrin gel containing the dextran (positive chamber) to an adjacent chamber initially dextran-free (negative chamber) under static or flow conditions. 

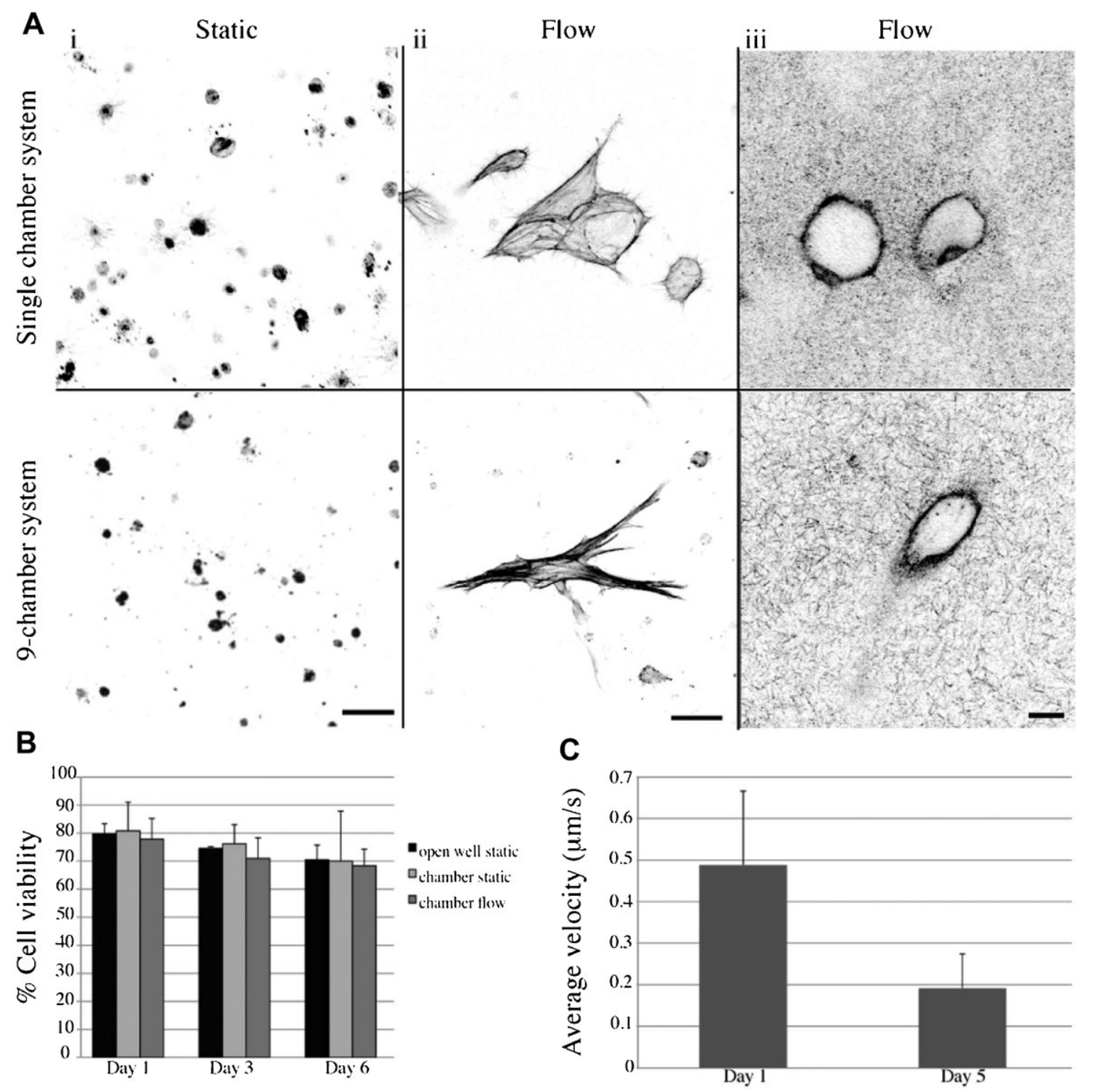

Figure 3. Lymphatic endothelial cell (LEC) capillary formation in response to flow after 5 days comparing a single chamber system with the nine-chamber system as seen by actin (phalloidin) staining and confocal reflectance followed by image inversion. A: (i) In both chamber models, LECs remain rounded in static conditions. (ii) Under flow conditions, LECs organize into multicellular structures. (i) and (ii) show projections of four equal thickness $(65 \mu \mathrm{m}) 3 \mathrm{D}$ stacks. (iii) As determined by confocal reflectance in a single $z$-slice, these structures contain lumen. Scale bars: $\mathrm{i}-\mathrm{ii}, 50 \mu \mathrm{m} ; \mathrm{iii}, 15 \mu \mathrm{m}$. B: Cell viability compared in an open well, under flow conditions or under static conditions. Viability is similar in all cases, and decreases by only about $10 \%$ over 5 days. C: Average interstitial flow velocities in the same chambers at the beginning and end of the 5 -day experiment. The average flow decreases, which is consistent with increasing cell volume.

that was fully exposed to culture medium (i.e., the cell-gel mixture in a 24 -well plate) and cells in our chambers, under both flow and static conditions. The viability decreased by approximately $10 \%$ over 5 days (Fig. 3B). We also compared FRAP measurements at the beginning and the end of an experiment and found that over 5 days, the average interstitial velocity in the mid-radius of the chamber decreased by more than half (Fig. 3C), reflecting overall matrix remodeling.

The structures that formed appeared relatively uniform within each gel. We also ran an experiment under reversed flow (i.e., flow from outlet to inlet). Due to the geometry of the system, under this condition it is only possible to reach a pressure gradient of $2 \mathrm{~mm} \mathrm{H}_{2} \mathrm{O}$ as compared to $5 \mathrm{~mm} \mathrm{H}_{2} \mathrm{O}$ in the other direction. Structure density was the same whether comparing the inner and outer half of the gel or different quadrants (Fig. 4), eliminating the question of nutrient limitation. This uniformity validated the use of the model for parallel studies.

We then compared LEC and BEC morphogenesis under flow versus $100 \mathrm{ng} / \mathrm{mL}_{2} \mathrm{PI}_{1-8}$ - $\mathrm{VEGF}_{121}$ and found yet more organization when exposed to the combined biophysical and 


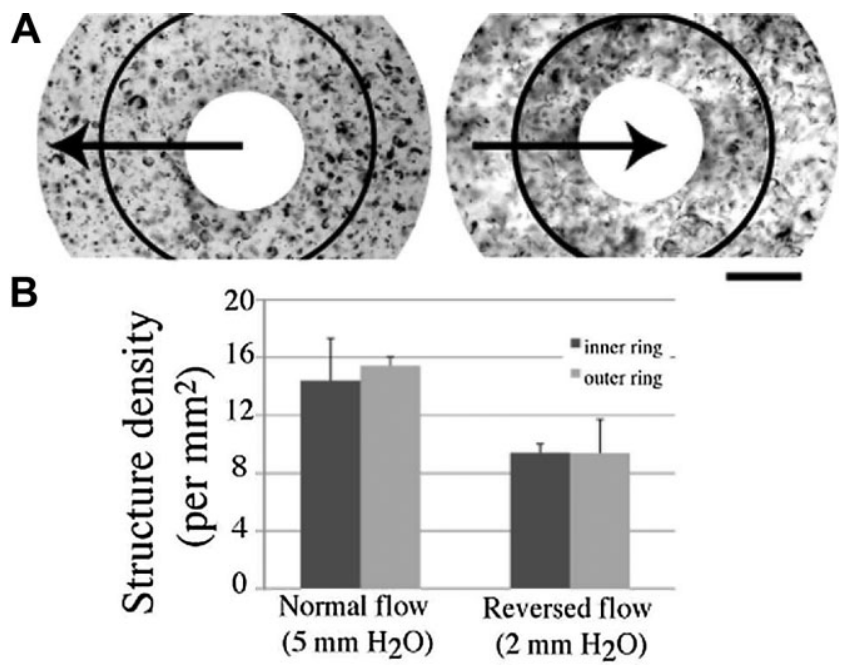

Figure 4. Overall homogeneity of cell organization is seen in all direction as well as between the inner and outer half of the gel. A: Shown are two chambers containing lymphatic endothelial cells after 5 days exposed to normal flow and reversed flow, respectively. Multicellular structures are seen throughout each chamber. Arrows indicate direction of flow. Scale bar: $200 \mu \mathrm{m}$. B: Quantification shows homogeneity, as the structure density is the same between both halves of the gel in normal or in reserved flow. The density in reversed flow conditions is lower due to the lower pressure head possible in this setup.

biomechanical cues, as previously reported (Helm et al., 2005). Specifically, when VEGF was added, the structures were more organized and denser (Fig. 5A). Quantitatively, the presence of VEGF in this case led to an increase of surface area covered by cell structures of $21 \%$ for LECs and $18 \%$ for BECs.

Consistent with their in vivo morphological differences, we saw that LECs formed more slender and elongated structures, while BECs were thicker and more circular in cross-section. In all four conditions, the structures contained lumen (Fig. 5B).

\section{Fibroblast-Tumor Co-cultures}

To model tumor invasion, we first co-cultured murine fibroblasts and tumor cells under flow, since flow was previously shown to affect each cell type alone ( $\mathrm{Ng}$ and Swartz 2003; Ng et al., 2005; Shields et al., 2007). The gel could withstand fibroblast contraction over 5 days and both cell types were seen spread out and healthy (Fig. 6A,B). Seeding fibroblasts without the $\mathrm{PE}$ ring resulted in gel contraction: after 5 days culture under flow conditions, the gel area was reduced to $50 \%$ of its original area. We then demonstrated an alternative model in which tumor cells were seeded in only the central portion of a fibroblastcontaining gel such that flow can move out of the tumor into the fibroblast culture (Fig. 6C). In this way, invasion into the stromal compartment could be mimicked (Fig. 6D). After 5 days under flow conditions due to $5 \mathrm{~mm} \mathrm{H}_{2} \mathrm{O}$ (Fig. 2B,C), the tumor cells could be seen invading the fibroblast- populated region of the gels. This highlights the usefulness of this model for a variety of applications.

\section{Discussion}

Incorporating well-defined interstitial flow into tissueengineered constructs has not yet become common practice, largely due to the lack of robust model systems. In the present work, we developed a new multichamber fluidic system to model cell morphogenesis and cell-cell interactions in a 3D environment under physiological levels of interstitial flow and to test, side-by-side, how these processes are influenced by different microenvironments. This is possible because nine different experimental conditions can be compared under uniform external conditions (i.e., pressure drop) within a single experiment, or replicates for fewer conditions.

As example applications of our system, we verified that interstitial flow stimulates LECs and BECs to form multicellular structures with lumen, and furthermore that flow synergizes with matrix-bound VEGF to drive more robust capillary formation as previously demonstrated in more cumbersome, single-chamber systems (Helm et al., 2005; Ng et al., 2004). Indeed, our device has many features which could prove useful to those studying angiogenesis and lymphangiogenesis, as the effects of different growth factors or inhibitors on cell morphogenesis and structure formation can be compared, as well as synergistic effects between flow and signaling molecules, which we demonstrated here (Fig. 5). Furthermore, the device is useful for a range of applications; for example, the model is advantageous for long-term culture of contractile cells like fibroblasts, unlike other flow models that use PDMS pillars as a gel boundary (Ng and Pun, 2008; Vickerman et al., 2008), which do not prevent contraction. We also showed that interactions between two cell types can be observed, in which one cell type (e.g., fibroblasts) can be seeded in the entire chamber and another cell type (e.g., tumor cells) can be injected in the inlet. Invasion of the tumor cells into the fibroblast region can be monitored by time-lapse imaging, cell morphology and changes in organization can be visually observed, and the gels can be extracted to analyze the factors secreted by the cells, in the absence or presence of flow. Different concentrations of collagen or fibrin or incorporation of factors into the matrix can be tested on cells, and cell-matrix interactions can be visualized using techniques like confocal reflectance microscopy (as shown in this study) or second harmonic generation via multiphoton microscopy.

This multichamber fluidic device has many advantages over other systems. First, it is suitable for long-term 3D cultures. Second, by reducing the size of the chamber, we not only reduced the amount of materials and cells needed by 10 -fold, but also decreased the diffusion length which is important for static controls to receive nutrients from the medium. Given that the length from the inlet to the outlet is $5.55 \mathrm{~mm}$ in the single radial flow system and only $2.25 \mathrm{~mm}$ 


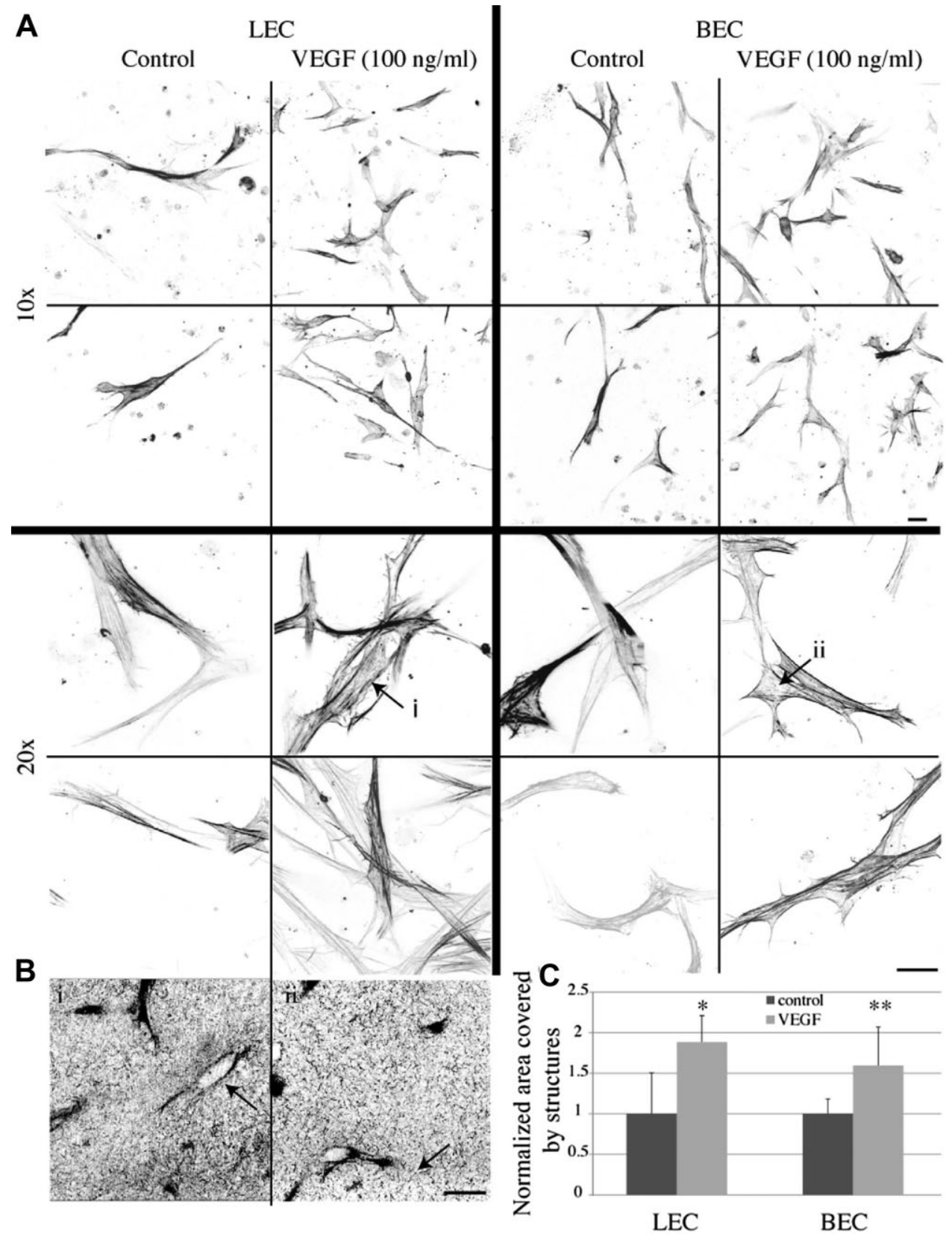

Figure 5. Combined effects of flow and $\alpha_{2} \mathrm{Pl}_{1-8}-\mathrm{VEGF}_{121}$ on lymphatic and blood endothelial cell (LEC and BEC) organization. A: With flow alone, cells formed multicellular structures, but in the presence of $\alpha_{2} \mathrm{Pl}_{1-8^{-}}-\mathrm{VEGF}_{121}$, structure density increased and vessel morphology improved. Also, more sprouting was seen in $\mathrm{BEC}$ cultures with $\alpha_{2} \mathrm{Pl}_{1-8^{-}}$ VEGF $_{121}$ than with flow alone. Shown are 2D projections of 3D confocal stacks using actin (phalloidin) for visualization followed by image inversion; scale bars: low magnification, $50 \mu \mathrm{m}$; high magnification, $20 \mu \mathrm{m}$. B: Confocal reflectance slice shows that both types of cell structures (i, LECs and ii, BECs) formed in the presence of $\alpha_{2} \mathrm{Pl}_{1-8}-\mathrm{VEGF}_{121}$ and flow form lumen. Scale bar: $20 \mu \mathrm{m}$. C: Quantification of cell spreading as the area fraction occupied by cells demonstrates that the presence of $\alpha_{2} \mathrm{PI}_{1-8}-\mathrm{VEGF}_{121}$ increases total structure formation both for LECs and BECs $(P<0.05$ and $<0.02$, respectively).

in the nine-chamber radial flow system, the time required for a nutrients to diffuse from the inlet to the center of the distance between the inlet and the PE ring is six times lower in the nine-chamber radial flow system compared to the previous single radial flow system, allowing better static controls to be made. Furthermore, the surface to volume ratio is two and a half times greater in the nine-chamber system, once again allowing better nutrient delivery. The small size also allows the nine chambers of the system to fit on a traditional microscope stage, which together with the good optical properties of PDMS, is ideal for both real-time observation and post-fixation microscopy (with fixation and staining performed directly in the intact chamber). Each chamber has a predictable and relatively homogeneous flow 

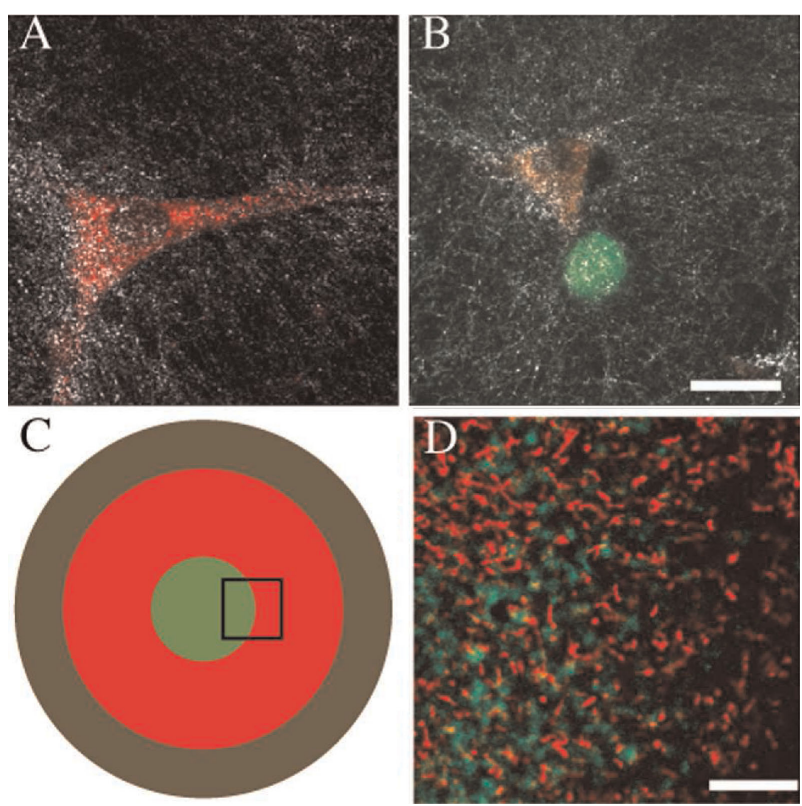

Figure 6. Demonstration of two types of tumor microenvironments in the flow chambers. A,B: Live confocal reflectance and fluorescence microscopy reveals cell and matrix organization after 2 days under flow conditions of $(A)$ human dermal fibroblasts (red) or (B) co-culture of the fibroblast with GFP-MDA-MB-435 carcinoma cells (green). After 2 days, HDF had spread and presumably imposed stress on the matrix, but no overall gel contraction or detachment was seen, even after 6 days of culture. Scale bar: $20 \mu \mathrm{m}$. C: A second tumor microenvironment model possible with the radial flow chambers consists of a fibroblast-populated gel with tumor cells injected into the center to form a central mass. D: Mouse 3T3 fibroblasts (red) were uniformly seeded in the gel, and then GFP-B16-F10 murine melanoma cells (green) were injected into the inlet at high density $\left(3 \times 10^{6} \mathrm{cells} / \mathrm{mL}\right)$. The tumor cells can be seen invading the gel after 7 days of culture under flow conditions. This image corresponds to the black box in (C). Scale bar: $200 \mu \mathrm{m}$.

pattern, as shown by the FRAP data and derived flow profiles, which is similar to what would be predicted from the chamber's radial geometry (Fig. 2). These flow characteristics can be tuned by adjusting the applied pressure head (Fig. 2), allowing us to achieve interstitial flow velocities ranging from less than $0.1 \mu \mathrm{m} / \mathrm{s}$ to more than $2 \mu \mathrm{m} / \mathrm{s}$, covering the entire physiological range and into pathophysiological values, such as may occur in inflammation or during tumor progression (Chary and Jain, 1989). Not all cells formed structures, but they grew homogeneously through a chamber as well as the chambers of a single system (Fig. 4), which further demonstrates that flow is present through the chambers in the flow condition but not in the static condition, and that flow is homogeneous within the system. We also found that the bond between the PDMS and the glass coverslip is reversible and the gel can be easily removed (although not in an intact state), allowing cell extractions (between 100,000 and 120,000 cells per gel) and assays such as RT-PCR and Western blots to be performed.

A potential pitfall of our system is its shared inlet and outlet medium reservoirs. While this was designed to ensure equal pressure drops across each chamber, there could be concern about contamination of soluble factors from one chamber to another via diffusion, if diffusive transport on the length and time scales of the model were greater than the convection that would counteract it. For a $5 \mathrm{~mm}$ pressure difference, flow varies from $\sim 2 \mu \mathrm{m} / \mathrm{s}$ in the inlet to $\sim 0.5 \mu \mathrm{m} / \mathrm{s}$ in the outlet. The distance between wells is $10 \mathrm{~mm}$ at the inlet and $16 \mathrm{~mm}$ at the outlet, and for soluble factors with diffusion coefficients on the order of $10^{-6}$ $10^{-7} \mathrm{~cm}^{2} / \mathrm{s}$ (Swartz, 2007, \#66), Peclet numbers are on the order of $80-1,000$. This means that for most proteins and other secreted factors, convection will counteract diffusion by 1-3 orders of magnitude and make cross-contamination effects very small. This was confirmed with our tests using $10 \mathrm{kDa}$ rhodamine-dextran (Fig. 2D), which showed that cross-contamination, particularly under flow conditions, was likely to be minimal, and even more so if active proteins are matrix-bound, as in the case of the $\alpha_{2} \mathrm{PI}_{1-8}-\mathrm{VEGF}_{121}$ used here. Nonetheless, diffusion is to be considered when comparing different environments, and to further decrease chances of contamination, different conditions can be seeded in opposing chambers rather than adjacent. Finally, since diffusing molecules are removed when changing the culture medium, exhaustion of growth factors could be a limiting factor to the maximum length of experiment, as well as matrix degradation.

The effects of interstitial flow on angiogenesis and lymphangiogenesis are significant: it helps endothelial cells grow and organize, directs them to migrate and delivers molecules to or creates gradients around cells. Interstitial flow has similarly significant effects in many other biological systems as well, including tumor cell migration (Shields et al., 2007) and fibroblast differentiation and matrix remodeling ( $\mathrm{Ng}$ and Swartz, 2003, 2006; $\mathrm{Ng}$ et al., 2005). Incorporating interstitial flow as a core design principle in tissue engineering represents an important step toward recapitulating the physiological environment of tissues, and our multichamber model is an important milestone toward achieving better mimicking the in vivo environment of cells using rationally designed 3D fluidic devices. Our multichamber fluidic system for maintaining long-term 3D cultures in the presence of interstitial flow is robust and versatile, allowing us to ask questions about how interstitial flow affects tissue morphogenesis and how other aspects of the microenvironment-cells, extracellular matrix, cytokines, and growth factors - are affected by flow to promote tissue formation and regeneration. Such approaches have the promise of opening new avenues of inquiry and novel approaches to engineering functional tissues.

The authors are grateful to Didier Foretay and Esra Güc for helpful advice and assistance, Alice Tomei for the murine cells and assistance, Mihaela Skobe for the MDA-MB-435 cells, Didier Trono for the pgkGFP lentiviral vector, Sanjiv Luther for the lymph node fibroblast reticular cells, and Andreas Zisch for the $\alpha_{2} \mathrm{PI}_{1-8}-\mathrm{VEGF}_{121}$. This work was funded in part by the European Framework Project 7 (NMP2007-LARGE) and the Susan G. Komen Foundation. 


\section{References}

Berk DA, Swartz MA, Leu AJ, Jain RK. 1996. Transport in lymphatic capillaries. II. Microscopic velocity measurement with fluorescence photobleaching. Am J Physiol 270(1 Pt 2):H330-H337.

Boardman KC, Swartz MA. 2003. Interstitial flow as a guide for lymphangiogenesis. Circ Res 92(7):801-808.

Buschmann MD, Kim YJ, Wong M, Frank E, Hunziker EB, Grodzinsky AJ. 1999. Stimulation of aggrecan synthesis in cartilage explants by cyclic loading is localized to regions of high interstitial fluid flow. Arch Biochem Biophys 366(1):1-7.

Chary SR, Jain RK. 1989. Direct measurement of interstitial convection and diffusion of albumin in normal and neoplastic tissues by fluorescence photobleaching. Proc Natl Acad Sci USA 86(14):5385-5389.

Evans RC, Quinn TM. 2006. Dynamic compression augments interstitial transport of a glucose-like solute in articular cartilage. Biophys J 91(4): 1541-1547.

Fleury ME, Boardman KC, Swartz MA. 2006. Autologous morphogen gradients by subtle interstitial flow and matrix interactions. Biophys $J$ 91(1):113-121.

Griffith LG, Swartz MA. 2006. Capturing complex 3D tissue physiology in vitro. Nat Rev Mol Cell Biol 7(3):211-224.

Hayward LN, Morgan EF. 2009. Assessment of a mechano-regulation theory of skeletal tissue differentiation in an in vivo model of mechanically induced cartilage formation. Biomech Model Mechanobiol [Epub ahead of print].

Helm CL, Fleury ME, Zisch AH, Boschetti F, Swartz MA. 2005. Synergy between interstitial flow and VEGF directs capillary morphogenesis in vitro through a gradient amplification mechanism. Proc Natl Acad Sci USA 102(44):15779-15784.

Helm CL, Zisch A, Swartz MA. 2007. Engineered blood and lymphatic capillaries in 3-D VEGF-fibrin-collagen matrices with interstitial flow. Biotechnol Bioeng 96(1):167-176.

Kolodney MS, Wysolmerski RB. 1992. Isometric contraction by fibroblasts and endothelial cells in tissue culture: A quantitative study. J Cell Biol 117(1):73-82.

Ng CP, Pun SH. 2008. A perfusable 3D cell-matrix tissue culture chamber for in situ evaluation of nanoparticle vehicle penetration and transport. Biotechnol Bioeng 99(6):1490-1501.

$\mathrm{Ng}$ CP, Swartz MA. 2003. Fibroblast alignment under interstitial fluid flow using a novel 3-D tissue culture model. Am J Physiol Heart Circ Physiol 284(5):H1771-H1777.

Ng CP, Swartz MA. 2006. Mechanisms of interstitial flow-induced remodeling of fibroblast-collagen cultures. Ann Biomed Eng 34(3): $446-454$.
Ng CP, Helm CL, Swartz MA. 2004. Interstitial flow differentially stimulates blood and lymphatic endothelial cell morphogenesis in vitro. Microvasc Res 68(3):258-264.

Ng CP, Hinz B, Swartz MA. 2005. Interstitial fluid flow induces myofibroblast differentiation and collagen alignment in vitro. J Cell Sci 118(Pt 20):4731-4739.

Pedersen JA, Swartz MA. 2005. Mechanobiology in the third dimension. Ann Biomed Eng 33(11):1469-1490.

Rutkowski JM, Boardman KC, Swartz MA. 2006. Characterization of lymphangiogenesis in a model of adult skin regeneration. Am J Physiol Heart Circ Physiol 291(3):H1402-H1410.

Santos A, Bakker AD, Zandieh-Doulabi B, Semeins CM, Klein-Nulend J. 2009. Pulsating fluid flow modulates gene expression of proteins involved in Wnt signaling pathways in osteocytes. J Orthop Res 27: 1280-1287.

Shields JD, Fleury ME, Yong C, Tomei AA, Randolph GJ, Swartz MA. 2007. Autologous chemotaxis as a mechanism of tumor cell homing to lymphatics via interstitial flow and autocrine CCR7 signaling. Cancer Cell 11(6):526-538.

Skobe M, Hawighorst T, Jackson DG, Prevo R, Janes L, Velasco P, Riccardi L, Alitalo K, Claffey K, Detmar M. 2001. Induction of tumor lymphangiogenesis by VEGF-C promotes breast cancer metastasis. Nat Med 7(2):192-198.

Swartz MA, Fleury ME. 2007. Interstitial flow and its effects in soft tissues. Annu Rev Biomed Eng 9:229-256.

Swartz MA, Hubbell JA, Reddy ST. 2008. Lymphatic drainage function and its immunological implications: From dendritic cell homing to vaccine design. Semin Immunol 20(2):147-156.

Tomei AA, Siegert S, Britschgi MR, Luther SA, Swartz MA. 2009. Fluid flow regulates stromal cell organization and CCL21 expression in a tissueengineered lymph node model. J Immunol 183(7):4273-4283.

Vickerman V, Blundo J, Chung S, Kamm R. 2008. Design, fabrication and implementation of a novel multi-parameter control microfluidic platform for three-dimensional cell culture and real-time imaging. Lab Chip 8(9):1468-1477.

Wang S, Tarbell JM. 2000. Effect of fluid flow on smooth muscle cells in a 3-dimensional collagen gel model. Arterioscler Thromb Vasc Biol 20(10):2220-2225.

Yong C, Bridenbaugh EA, Zawieja DC, Swartz MA. 2005. Microarray analysis of VEGF-C responsive genes in human lymphatic endothelial cells. Lymphat Res Biol 3(4):183-207.

Zisch AH, Schenk U, Schense JC, Sakiyama-Elbert SE, Hubbell JA. 2001. Covalently conjugated VEGF-fibrin matrices for endothelialization. J Control Release 72(1-3):101-113. 